\title{
FORESTACIÓN MULTIPROPÓSITO PARA LA REGIÓN DE AYSÉN
}

Cisternas, Juan Carlos ${ }^{1}$

\section{RESUMEN}

La necesidad de conciliar políticas sectoriales, metas institucionales e intereses particulares en la región de Aysén, torna compleja e incierta la decisión de establecer plantaciones forestales, particularmente si sus objetivos no recogen la pertinencia cultural con sus habitantes.

A través de una exhaustiva identificación, basada en atributos autoecológicos y homologaciones edafoclimáticas se seleccionaron dos especies nativas para forestar en Aysén con objetivos multipropósito dados por que efectivamente constituyan masa forestal, que puedan conciliar la producción ganadera y forestal sin necesidad de exclusión animal y que cumplan funciones ambientales en suelos que carecen de cobertura forestal.

La selección de especies fue contrastada con la evaluación de procesos de colonización natural que se ha estado produciendo en algunos sectores de transición hacia estepa patagónica; con experiencias de plantaciones pilotos y forestaciones tradicionales en el marco del DL 701 . Las especies seleccionadas son araucaria (Araucaria araucana) y radal (Lomatia hirsuta).

De las observaciones levantadas en terreno, se concluye que araucaria puede ser establecida a una densidad de 700 plantas por hectárea, con presencia de una carga animal del tipo ovino o bovino, no superior a 0,5 y 2 unidades por hectárea, respectivamente. No ocurre daño por ramoneo y el daño mecánico por efecto del pisoteo no supera el $5 \%$ de las plantas. En este caso se concilia la producción maderera, no maderera y ganadera.

Radal puede ser establecida en laderas con avanzados procesos de erosión y con presencia de animales del tipo ovino y bovino. La especie cumple la función de protección al suelo, producción dendroenergética, abrigo a los animales, efecto nodriza para la regeneración de especies mas exigente. Concilia la producción ganadera, maderera y protección.

Palabras Claves: Forestación multipropósito; Selección especies, Araucaria, Radal

\section{SUMMARY}

The need to harmonize sectorial policies, institutional goals and particular interests in Aysen's region, turns the decision to establish forest plantations in complex and uncertain; particularly, if the aim is not gathering the cultural pertinence to the inhabitants.

Through an exhaustive identification based on autoecological attributes and edafoclimátic homologations, two native species were selected for afforestation in the region with multipurpose

1 Corporación Nacional Forestal. Chile. juan.cisternas@conaf.cl 
objectives, including that they really can constitute a forest mass without cattle exclusion, they can harmonize the cattle and forest production without the need of cattle exclusion and they fulfill environmental functions in soils currently without forest cover.

Species selection was confirmed by evaluation of natural processes that has been taking place in some sectors of transition towards Patagonian steppe and taking in due account local experiences with pilot plantations and traditional afforestation on the frame of the DL 701. The selected species were Araucaria (Araucaria araucana) and Radal (Lomatia hirsuta).

Over the basis of field data revision it is concluded that araucaria can be established to a density of 700 plants by hectare, with presence of ovine o bovine cattle, not higher than 0.5 and 2 units by hectare, respectively. There is no hurt by lop, mechanical damage over the plants is not higher than $5 \%$ and can be combined that way wood, non wood and cattle production

Radal can be established in hillsides with serious erosion processes and with the presence of ovine or bovine cattle. The species fulfills a variety of functions, including soil protection, fuel wood production, cattle shelter and wet nurse effect for other more site demanding species, allowing that way wood, non wood and cattle production and also soil protection.

Keywords: Multipurpose forestry, Species selection, Araucaria, Radal

\section{INTRODUCCIÓN}

La región de Aysén debe apostar por diversificar los objetivos de la forestación. Se han realizado y se continúan estableciendo plantaciones con objetivos principalmente orientados a la producción de pulpa, pero su rentabilidad es dudosa y esto lo demuestra el hecho que existiendo más de un millón de hectáreas disponibles para forestar en la región, sólo se han plantado alrededor de 45 mil hectáreas durante los últimos 20 años.

A los tradicionales objetivos de la forestación (producción maderera y protección), deben incorporarse objetivos contemporáneos y con pertinencia cultural; bonos de carbono, dendroenergía, agroforestería, producción no maderera, restauración ecológica, plantaciones mixtas multipropósitos, formación de masas boscosas en interfase urbano-rural, y otros.

La diversificación también debe darse en el ámbito de las especies. Para evitar tendencias hacia monocultivos y sus eventuales consecuencias contraproducentes, surge la necesidad de evaluar alternativas técnicamente factibles y financieramente atractivas para los propietarios de la región, que permitan lograr la diversificación planteada.

Resulta indispensable identificar especies, especialmente nativas, con atributos económicos, sociales y ambientales, que se acomoden a las condiciones ambientales de la región, y encajen con el acervo cultural del mundo rural de la Patagonia chilena. 


\section{OBJETIVOS}

El objetivo de este trabajo es destacar, a través de estudios de caso, dos especies nativas con las cuales incentivar la diversificación y complementar los objetivos de la forestación en la región de Aysén, especies que además respondan a los intereses particulares de los propietarios, y socio - ambientales del país.

Como objetivos específicos se pueden indicar los siguientes:

Identificar dos especies nativas con potencial (productivo, social o ambiental), para forestar en la región de Aysén.

Describir las características autoecológicas de las especies identificadas.

Entregar antecedentes de establecimiento, sobrevivencia, crecimiento y objetivos de forestación para estas especies.

\section{MATERIAL Y MËTODO}

Se efectuó una identificación y selección de especies nativas, que por sus requerimientos autoecológicos, ambientales y atributos económicos y sociales, pudiesen ser de interés para incentivar las plantaciones en la región de Aysén.

La principal característica que debían tener las especies candidatas, es que pudiesen establecerse y desarrollarse con presencia de animales domésticos.

Analizando la base de datos de CONAF, se identificaron plantaciones con especies acreditadas y se les hizo un seguimiento para determinar el comportamiento en cuanto a sobrevivencia y desarrollo, durante los primeros años. Posteriormente, se hizo un chequeo en terreno de las más promisorias para levantar información dasométrica y recoger de primera fuente la opinión de los propietarios respecto de plantaciones experimentales con especies no tradicionales.

De todas las especies revisadas, araucaria (Araucaria araucana), fue la única que cumplía ese requisito y que además otorgaba la posibilidad de combinar la producción animal (engorda - lana - crianza), con la producción forestal (madera de alta calidad y frutos). La selección de radal (Lomatia hirsuta), se dio en forma casual, al observar el comportamiento de esta especie que se encuentra colonizando, con presencia de animales domésticos del tipo bovino, campos agrícolas abandonados en el sector El Maitenal de Lago Verde, comuna del mismo nombre. 


\section{RESULTADOS}

\section{Araucaria}

\section{-Clima y Suelo}

El clima característico varía entre templado cálido, representado por veranos secos y precipitaciones concentradas en invierno en forma de nieve, a templado con una marcada influencia mediterránea y hasta 4 meses secos durante el verano.

La precipitación, dependiendo de la altitud y longitud, varía entre $800 \mathrm{~mm}$ al año (estepa argentina) hasta $4000 \mathrm{~mm}$ anuales con parte de ella en forma de nieve (vertiente occidental de la Cordillera de Los Andes). Las temperaturas medias fluctúan entre $-5^{\circ} \mathrm{C}$ (con extremos de $-20^{\circ} \mathrm{C}$ ) en invierno, hasta los $30^{\circ} \mathrm{C}$ en verano (Di Castri y Hajek, 1976; Veblen, 1982; Veblen et al., 1995; Donoso, 2006).

Los suelos en donde se distribuye la especie han sido desarrollados sobre rocas volcánicas andesíticas y basálticas cuaternarias cubiertas por capas de cenizas volcánicas recientes, pumicitas y escoria volcánica. En la Cordillera de la Costa, se han desarrollado in situ de antiguas rocas graníticas y metamórficas del tipo micaesquistos.

La profundidad del suelo en que crece presenta importantes variaciones dependiendo de la topografía, pero en general son delgados a moderadamente profundos (de 15 a $180 \mathrm{~cm}$ ), de textura media a fina en la superficie y en profundidad franca arcillo arenosa. Son suelos de erodabilidad (susceptibilidad a la erosión), moderada a alta con drenaje rápido a moderado; $\mathrm{pH}$ ácido y bajo nivel nutricional (Donoso et al., 1984; Donoso, 1993; Cortes, 2003).

\section{-Supervivencia y Crecimiento en Plantaciones}

Los antecedentes documentados en relación a plantaciones de araucaria son escasos. De acuerdo a Donoso (2006), en la región de La Araucanía se concentran los mayores esfuerzos por forestar y restaurar sitios con esta especie, pero lamentablemente no existen controles o evaluaciones sobre su desarrollo y éxito de establecimiento. No obstante lo anterior, los pocos estudios documentados son bastante heterogéneos, derivado esto probablemente de las condiciones de sitio y tipo de plantas usados (raíz desnuda, en contenedor, siembra directa).

Estudios realizados en Misiones, Argentina, con pino Paraná (Araucaria angustifolia), señalan que los mejores resultados, en supervivencia y crecimiento, se logran con plantas en macetas de un año, plántulas en tubete o speedling de cinco meses y siembra directa (Kurtz et al., 2003). En la zona cordillerana de la región del Bio Bio se está plantando araucaria 1:0 en speedling con sobrevivencia sobre el $80 \%$ (Donoso, 2006).

En la región de Aysén, observaciones realizadas por el autor, dan cuenta de resultados disímiles. En La Junta, se realizó una plantación con araucaria a raíz desnuda en una superficie de 7 hectáreas con presencia de ganado ovino y bovino dentro de la plantación; al cabo de cuatro años, la supervivencia fue cercana al 95\%. (Figura $N^{\circ} 1$ ). El único daño reportado es 
por pisoteo y sólo ocurrió los dos primeros años. El propietario señaló que el sector se usa como invernada, con presencia de 5 a 7 vacunos entre los meses de mayo y septiembre. Los sectores protegidos por la cobertura de copas de araucarias, no son pastados, debido a que el tipo de ramoneo de los vacunos expone la lengua a la morfología foliar (coriácea terminada en un micrón agudo tipo aguja), por lo que los animales evitan su contacto.
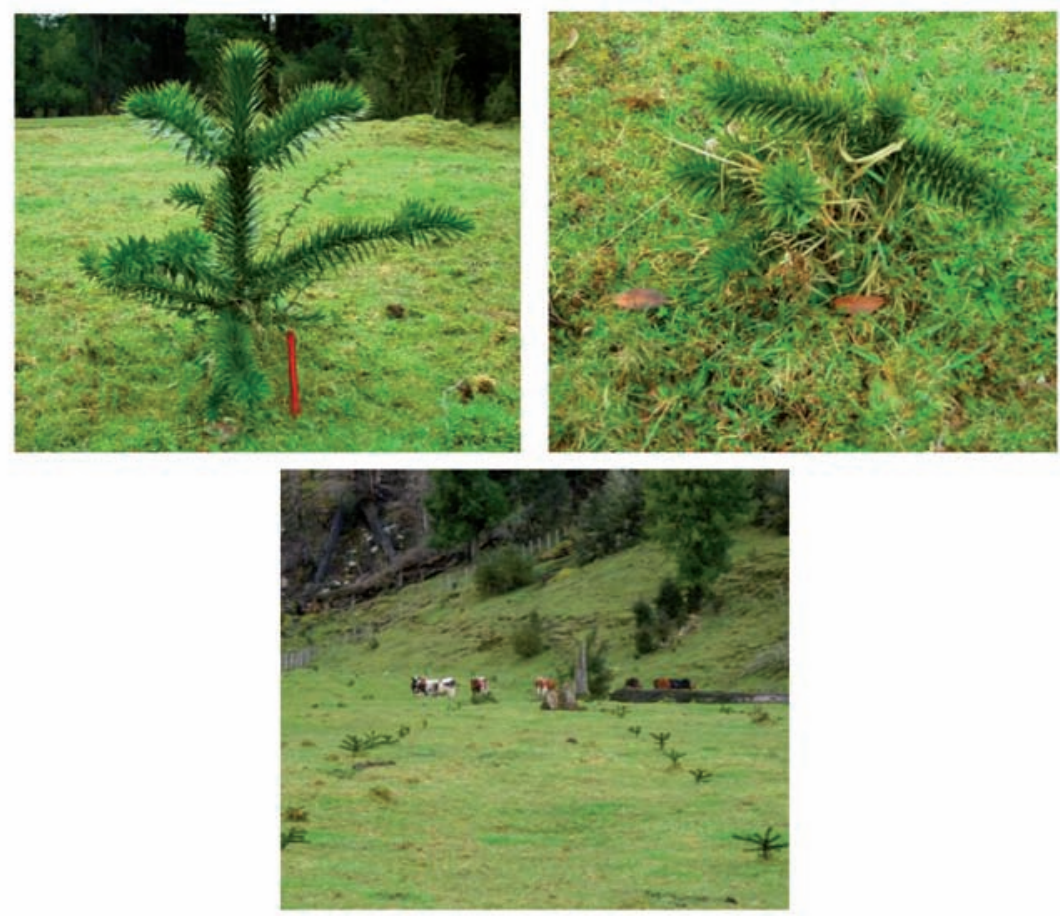

Figura $N^{\circ} 1$

\section{PLANTACIÖN DE ARAUCARIAS DE CUATRO AÑOS CON PRESENCIA DE BOVINOS}

Otra plantación similar (Figura $\mathrm{N}^{\circ} 2$ ), se realizó en el mismo sector con exclusión de ganado y al cabo del primer año se logró acreditar una supervivencia cercana al $82 \%$. Sin embargo, a partir del segundo año el pasto comenzó a ahogar las plantas, terminando por suprimirlas completamente. Al año siguiente se volvió a plantar una superficie de 14 hectáreas. Al cabo de un año se logró acreditar una supervivencia de 78\%. Después del segundo año, el propietario ingresó, al rodal, animales del tipo caprino, cuyo efecto se manifiesta en el escaso desarrollo de las plantas productos del ramoneo. En este caso, este tipo de animales arranca o corta con los dientes, por lo que la morfología foliar no lo afecta. 

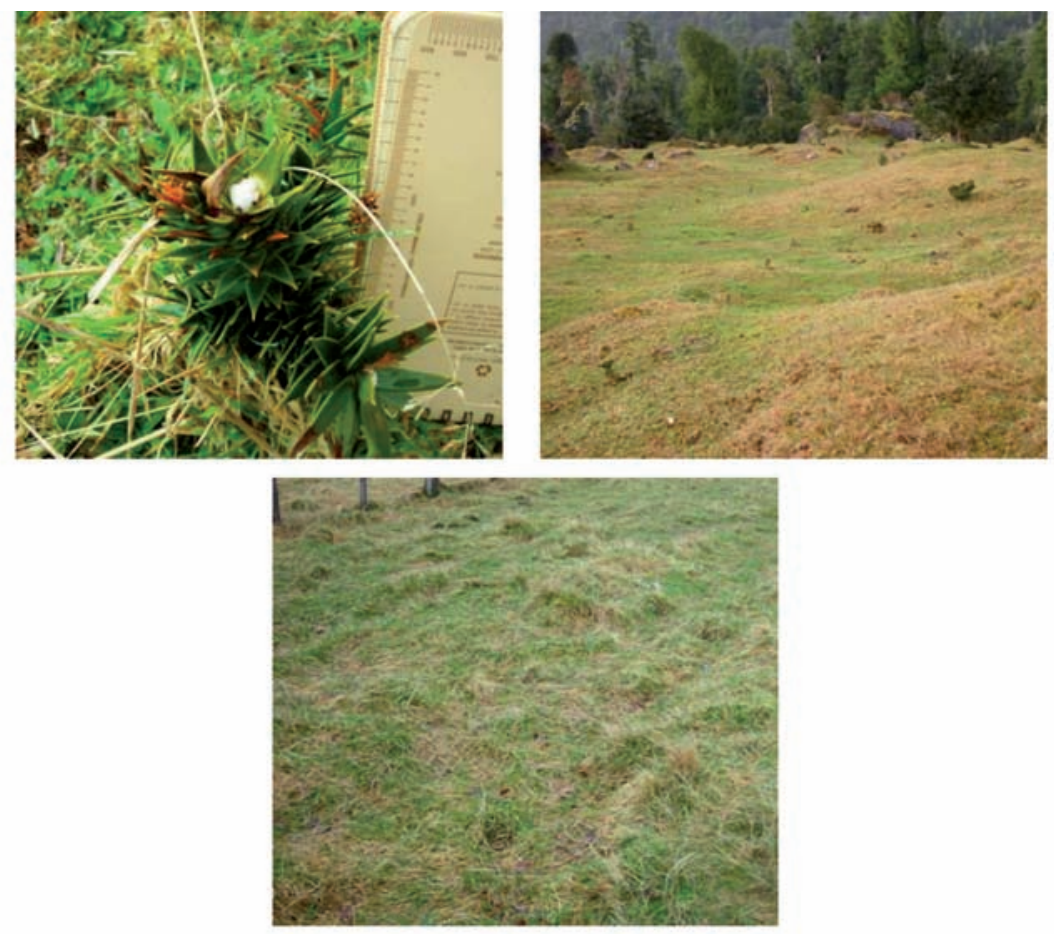

Figura $\mathrm{N}^{\circ} 2$

PLANTACIÖN DE ARAUCARIAS DE TRES AÑOS CON PRESENCIA DE CAPRINOS

Prendimientos superiores al $84 \%$ se lograron en tres plantaciones realizadas en el sector de La Tapera.

De igual modo se conocen antecedentes de una plantación realizada hace unos 10 años en la Reserva Nacional Lago Carlota, que fracasó rotundamente y en la actualidad sólo es posible encontrar algunos individuos cloróticos menores a 1 metro de altura y de mala forma.

En la localidad de Villa Ortega, se plantaron 3 hectáreas con escasa supervivencia; el replante fue exitoso sólo con plantas en contenedores.

También se sabe de forestaciones en el sector de Puerto Tranquilo (Provincia de General Carrera), con prendimientos sobre el $80 \%$ y otra de 6 hectáreas que fue afectada por sequía y cuya sobrevivencia fue de un $50 \%$.

En cuanto a crecimiento, Donoso (2006) menciona alturas de hasta $10 \mathrm{~m}$ y diámetros a la altura de pecho de hasta $20 \mathrm{~cm}$, en una siembra de 37 años en el Parque Nacional Tolhuaca. 
En Aysén, el autor, ha encontrado araucarias de 34 años de 18 metros de altura y $39 \mathrm{~cm}$ de diámetro a la altura de pecho (Figura $N^{\circ} 3$ ).
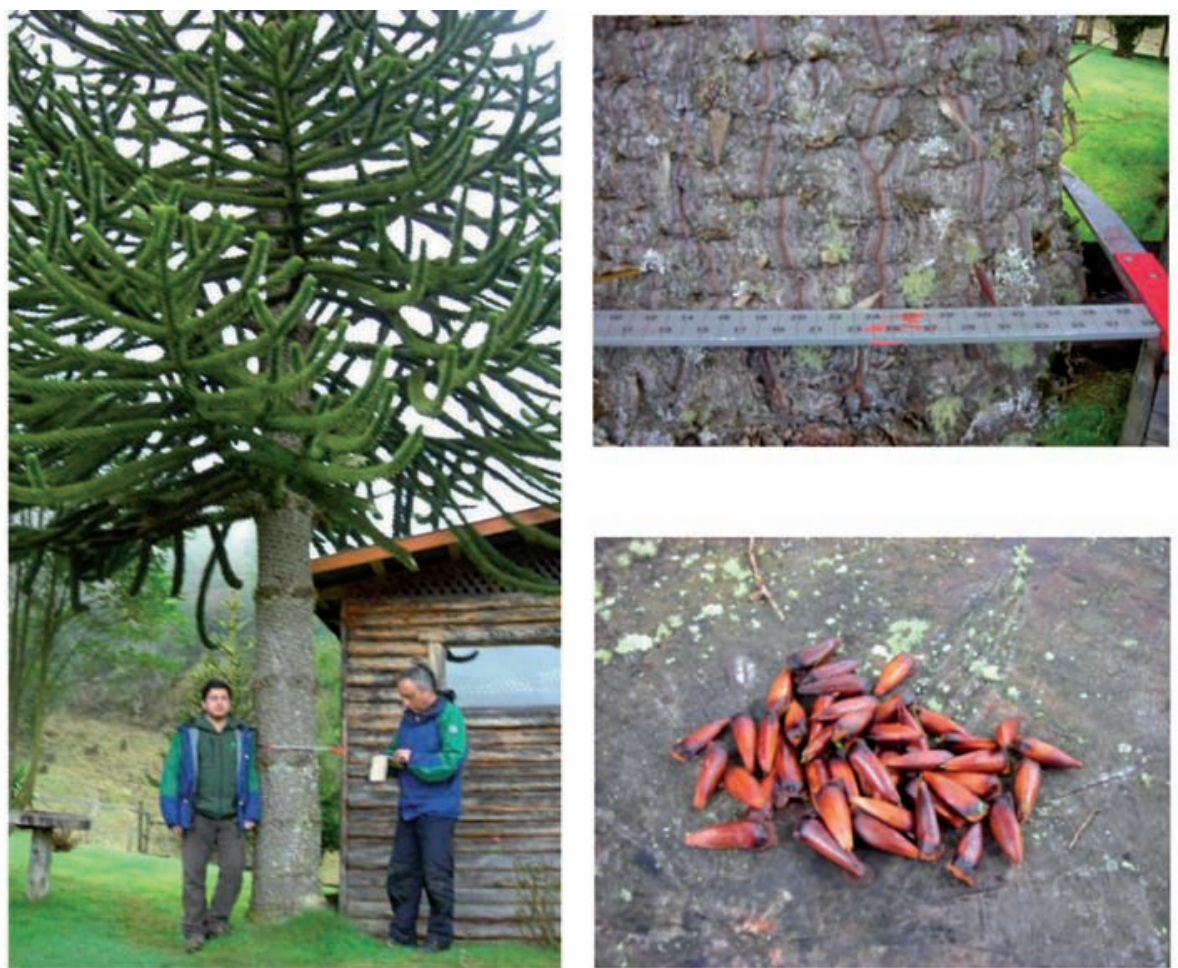

Figura $\mathrm{N}^{\circ} 3$

ARAUCARIAS DE 34 AÑOS CON PRODUCCIÖN DE PIÑONES, EN LA JUNTA

-Objetivos de la Forestación

Los objetivos de la forestación pueden variar desde la plantación tradicional de alta densidad (sobre los 1000 árboles por hectárea) para productos de alto valor comercial como madera aserrada y chapas, plantación de densidad intermedia (400 - 800 árboles por hectárea) orientada a la producción combinada y plantación de baja densidad con objetivos como protección, ornamentales o paisajísticos entre otros.

Si el objetivo es silvopastoral, se puede combinar la producción de carne, lana y crianza; la madera aserrada y chapas, y la producción de frutos. La prohibición de corta debido a su condición de Monumento Natural, quedó exenta por la Ley $\mathrm{N}^{\circ} 20.283$ cuando se trata de plantaciones no compensatorias.

Como ya se mencionó anteriormente, su follaje coriáceo no es palatable para animales 
domésticos del tipo ovino y bovino, por lo que no requiere de exclusión animal durante los primeros años de la plantación. Debe mencionarse si, que la carga animal en la plantación no debe ser mayor a una unidad de bovino por hectárea para no generalizar el daño mecánico por pisoteo. Lo anterior no aplica para el ganado del tipo caballar, que corta y arranca con los dientes su alimentación, y de caprino, que posee un labio superior muy movible insensible al mucrón unido a una composición salivar capaz de desactivar los taninos y por tanto dificultar el proceso de cicatrización de las especies ramoneadas.

\section{Cuadro $\mathrm{N}^{\circ} 1$ \\ OBJETIVOS DE LA FORESTACIÓN CON ARAUCARIA}

\begin{tabular}{|l|l|}
\hline Producción maderera & \\
\hline Producción de madera debobinada, chapas o aserrada & $\mathrm{x}$ \\
\hline Producción de tejuelas, varas o postes & $\mathrm{x}$ \\
\hline Producción de leña y carbón & $\mathrm{x}$ \\
\hline Producción no maderera & $\mathrm{x}$ \\
\hline Producción de fruto, flores, semillas, esencias, aceites, miel, otros & $\mathrm{x}$ \\
\hline Producción de follaje decorativo & \\
\hline Venta de bonos de carbono & \\
\hline Producción silvopastoral & $\mathrm{x}$ \\
\hline Producción de forraje & $\mathrm{x}$ \\
\hline Protección cortinas cortaviento, cercos vivos, galpones naturales & \\
\hline Producción combinada & \\
\hline Servicios ambientales & $\mathrm{x}$ \\
\hline Protección de agua, suelos, biodiversidad & $\mathrm{x}$ \\
\hline Realce belleza escénica, paisajismo & $\mathrm{x}$ \\
\hline Restauración ecosistemas & $\mathrm{x}$ \\
\hline Forestación urbana & \\
\hline
\end{tabular}

Su lento crecimiento natural es la principal desventaja para incentivar su masificación. Sin embargo, con los crecimientos reportados en La Junta, no es irreal pensar en rotaciones de 80 a 100 años, alturas de 30 a 40 m y diámetros sobre $50 \mathrm{~cm}$, bajo un esquema silvopastoral.

Otra desventaja es el riesgo potencial de ataque de roya, un hongo del género Mikronegeria que provoca la muerte del ejemplar afectado a través de la necrosis del tejido foliar. Este hongo necesita de la presencia de araucaria y de especies caducifolia del género Nothofagus para completar su circuito

\section{-Sitios a Forestar}

Las características climáticas y edáficas no serían limitantes para la supervivencia y desarrollo posterior de la especie en las provincias de Coyhaique y General Carrera (Cuenca del lago General Carrera). 
Las probabilidades de éxito son mayores en las partes altas de las cuencas hidrográficas (altitud superior a los $500 \mathrm{msnm}$ ) y en la vertiente oriental de la cordillera de Los Andes, con suelos algo desarrollados y medianamente profundos.

En la Provincia de Aysén, la competencia con las especies del Tipo Siempreverde y la rápida colonización de especies heliófitas en zonas descubiertas suponen una seria competencia que limitaría el establecimiento posterior de araucaria, particularmente en el territorio insular. La especie se establece y se desarrolla favorablemente en la parte continental, en sectores con suelos profundos y bien drenados. También su establecimiento inicial es exitoso aunque con menor crecimiento en suelos nutricionalmente pobres y que han perdido su cubierta vegetal, ya sea por perturbaciones naturales o antrópicas y en donde la competencia con especies del Tipo Siempreverde no se presenta.

\section{Radal}

Radal, es un árbol que puede alcanzar más de $20 \mathrm{~m}$ de altura y $80 \mathrm{~cm}$ de diámetro en las mejores condiciones de crecimiento, donde puede desarrollar un fuste relativamente recto y cilíndrico, de corteza delgada, de color gris o ceniza con manchas oscuras y claras alternadas. Sin embargo, su morfología habitual es en forma de renoval en estado monte bravo o latizal bajo, colonizando suelos perturbados, orillas de caminos y praderas abandonadas (Donoso, 2006). En la comuna de Lago Verde, se comporta como una especie colonizadora de rápido crecimiento inicial el que decae después de los 20 años en donde alcanza diámetros promedio de $13 \mathrm{~cm}$ y alturas de 8 a $10 \mathrm{~m}$ (Cisternas y Solís, 2009).

\section{-Clima y Suelo}

La distribución de radal muestra por si sola la gran variedad de condiciones climáticas en que ocurre. En la zona sur de su distribución se desarrolla dentro de las condiciones del clima oceánico templado húmedo en el lado occidental de la Cordillera de los Andes y dentro del clima templado seco en la vertiente oriental (Argentina).

En cuanto a suelo, no podría señalarse tipos de suelos específicos. Crece en suelos rocosos y áridos en la estepa hasta suelos trumaos profundos en la Cordillera de los Andes y suelos metamórficos variables en la Cordillera de la Costa.

\section{-Supervivencia y Crecimiento en Plantaciones}

Resultados obtenidos de un ensayo en invernadero (Donoso, 2006), indican que el inicio de la germinación ocurre treinta después de realizada la siembra. A cuarenta días de iniciada la germinación aparecieron las primeras hojas verdaderas. Luego, después de siete meses de la siembra, la altura promedio llegó a 2,4 cm. Al término de una temporada, las plantas alcanzaron una altura de $6,5 \mathrm{~cm}$. Luego del replante a platabanda y posterior a una nueva temporada de crecimiento (planta 1/1), es posible obtener plantas de 28 a $32 \mathrm{~cm}$ de altura en promedio.

En otro estudio, realizado en el predio Las Palmas de la Universidad Austral de Chile, se midieron alturas promedios de $6.9 \mathrm{~m}$ en altura y $9.3 \mathrm{~cm}$ de DAP, después de 11 años de 
establecida la plantación.

En la región de Aysén no hay antecedentes de plantaciones con radal. Su presencia es habitual en sectores en la vertiente oriental de Los Andes Patagónicos, particularmente en la comuna de Lago Verde, en donde se ha ido propagando masivamente (Cuadro $\mathrm{N}^{\circ} 2$ y Figura $N^{\circ} 4$ ), en forma natural a través de los cursos de agua (Cisternas y Solís, 2009).

En algunas regiones meridionales del país se usa la especie para leña a causa de su abundancia relativa en ciertos lugares, como la provincia de Palena o la región de Aysén, donde esta especie creció como renuevo después de que los bosques originales fueron incendiados a mediados del siglo veinte.

También tiene aprovechamientos no madereros. De la corteza y hojas, que contiene taninos, se extrae un colorante parar teñir de color café. La infusión de sus hojas inhibe la inflamación, además es apreciado como pectoral y antiespasmódico; hirviéndola con agua se obtiene un fuerte purgante (Wilhelm de Mösbach, 1999).
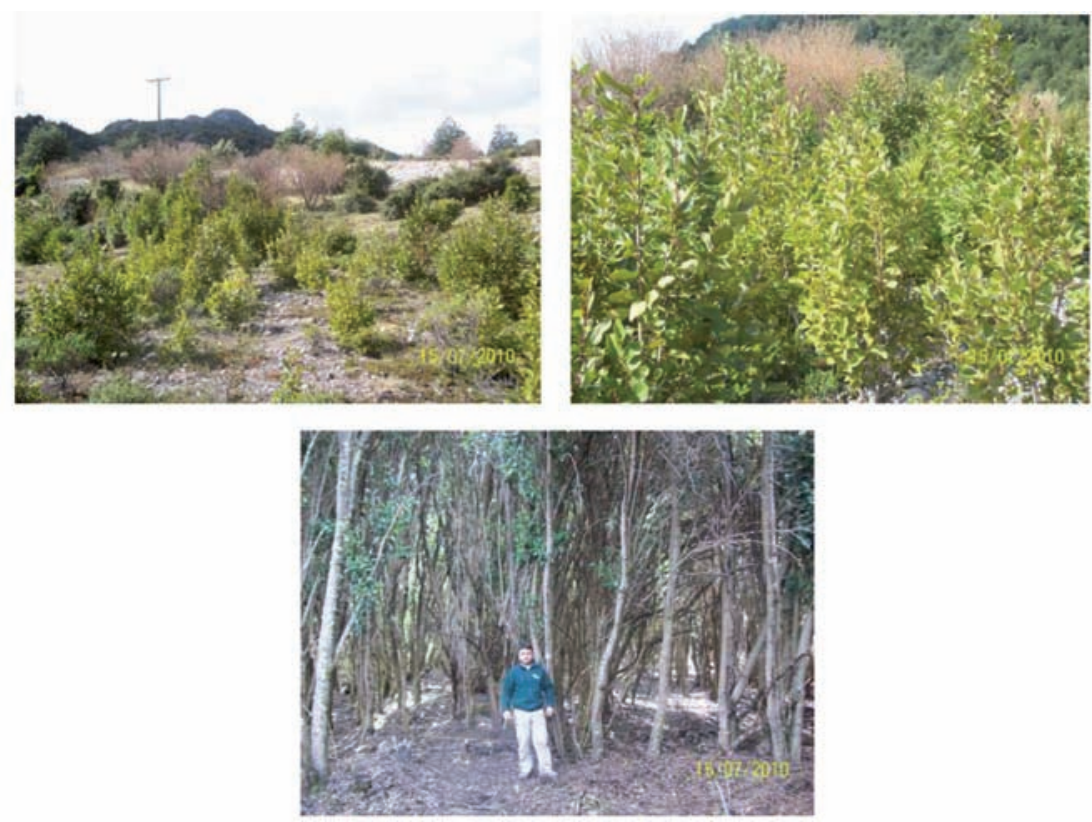

Figura $N^{\circ} 4$

ETAPAS DE DESARROLLO DE RADAL EN LAGO VERDE 
CUADRO N ${ }^{\circ} 2$

TABLA DE RODAL Y EXISTENCIAS DE RADAL EN LAGO VERDE

\begin{tabular}{|c|c|c|c|}
\hline $\begin{array}{c}\text { Clase DAP } \\
(\mathbf{c m})\end{array}$ & $\begin{array}{c}\text { Densidad } \\
(\mathbf{N} / \mathbf{h a})\end{array}$ & $\begin{array}{c}\text { Área Basal } \\
\left(\mathbf{m}^{2} / \mathbf{h a}\right)\end{array}$ & $\begin{array}{c}\text { Volumen Bruto } \\
\left(\mathbf{m}^{3} / \mathbf{h a}\right)\end{array}$ \\
\hline 7 & 800 & 3,82 & 20,42 \\
\hline 12 & 1.900 & 22,60 & 120,92 \\
\hline 17 & 1.300 & 27,76 & 148,48 \\
\hline 22 & 300 & 10,74 & 57,48 \\
\hline Total & 4.300 & 64,92 & 347,30 \\
\hline
\end{tabular}

\section{-Objetivos de la Forestación}

Debido a sus características de colonizador temprano, su gran capacidad de propagación, sus propiedades energéticas, su floración, y su no palatabilidad para el ganado doméstico, radal puede ser una especie altamente interesante para establecer plantaciones multipropósitos en la región de Aysén.

En suelos de aptitud forestal sometidos por más de 50 años a pastoreo directo en la localidad de Lago Verde en particular y en la región en general, el establecimiento inicial y rápida colonización de radal constituye una buena solución al proceso de erosión de suelos originado con la destrucción del bosque nativo primario aproximadamente en la década de 1930. Este comportamiento lo consigue gracias a la dispersión anemófila de sus semillas, su alta capacidad germinativa, su rápido crecimiento y su capacidad de reproducción vegetativa.

En las áreas en que se produce una fuerte alteración del bosque (especialmente en el tipo Siempreverde o bosques donde participa coigüe), puede formar bosquetes de segundo crecimiento o renovales.

En ausencia de nuevas perturbaciones, empiezan a surgir las especies que constituían el bosque original y los individuos de radal mueren o caen a medida a medida que van siendo superados en desarrollo y vigor por las otras especies, por lo tanto puede ser una especie interesante para restaurar bosques del Tipo Siempreverde o Lenga. 


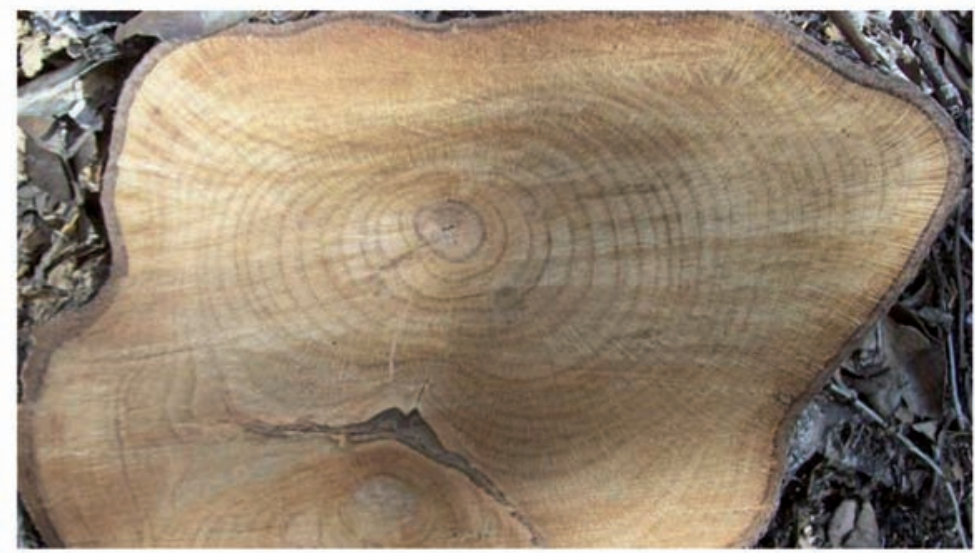

Figura $\mathrm{N}^{\circ} 5$

RADAL DE 25 AÑOS Y 28 CM DE DIÁMETRO

La madera del radal es altamente cotizada por su calidad, asimilándose en su aspecto y aplicación al nogal americano. Es liviana, elástica, de color gris y con vetas muy parecidas a las del notro y avellano. Es utilizada para mueblería, chapas y artesanía entre otros usos (Del Fierro et al., 1998). También se ha introducido su cultivo en España y Escocia como especie ornamental.

Según antecedentes empíricos otorgados por los propios pobladores de Lago Verde, el radal tiene regeneración de tocón vigorosa sólo en los estados juveniles, no así en bosque adulto, lo cual indica que radal es una especie pionera, temporal y facilitadora para el acceso de especies nativas más exigentes como ñirre, maitén y coihue en etapas posteriores.

La baja palatabilidad del follaje del radal respecto de las especie acompañantes (ñire, maitén, coigüe, coligüe), le otorga una ventaja adicional ya que no entra en conflicto con la ganadería de tipo extensivo que se realiza en la región y por tanto pueden coexistir perfectamente con unidades silvopastorales.

Debido a su alta producción de biomasa (Cuadro №2), con una producción volumétrica media superior a los $11 \mathrm{~m}^{3} / \mathrm{ha} / \mathrm{año}$ (superior en casi 100\% al crecimiento de lenga), puede ser interesante también desde el punto de vista dendroenergético. región.

En el Cuadro $N^{\circ} 3$ se indica los principales objetivos para la forestación con radal en la 


\section{Cuadro $\mathrm{N}^{\circ} 3$}

\section{OBJETIVOS DE FORESTACIÓN CON RADAL}

\begin{tabular}{|l|c|}
\hline Producción maderera & \\
\hline Producción de madera debobinada, chapas o aserrada & \\
\hline Producción de tejuelas, varas o postes & $\mathrm{X}$ \\
\hline Producción de leña y carbón & \\
\hline Producción no maderera & $\mathrm{X}$ \\
\hline Producción de fruto, flores, semillas, esencias, aceites, miel, otros & \\
\hline Producción de follaje decorativo & $\mathrm{X}$ \\
\hline Venta de bonos de carbono & \\
\hline Producción silvopastoral & $\mathrm{X}$ \\
\hline Producción de forraje & $\mathrm{X}$ \\
\hline Protección cortinas cortaviento, cercos vivos, galpones naturales & \\
\hline Producción combinada & $\mathrm{X}$ \\
\hline Servicios ambientales & $\mathrm{X}$ \\
\hline Protección de agua, suelos, biodiversidad & $\mathrm{X}$ \\
\hline Realce belleza escénica, paisajismo & $\mathrm{X}$ \\
\hline Restauración ecosistemas & \\
\hline Forestación urbana & \\
\hline
\end{tabular}

\section{-Sitios a Forestar}

Las características climáticas y edáficas no serían limitantes para la supervivencia y desarrollo posterior de la especie en las provincias de Coyhaique y General Carrera. Su establecimiento inicial puede ser en suelos nutricionalmente pobres y que han perdido su cubierta vegetal, ya sea por perturbaciones naturales o antrópicas y en donde la competencia con especies del Tipo Siempreverde no se presenta en las etapas iniciales de colonización.

También puede ser usada como especie nodriza que facilite el acceso, en forma natural o a través de plantaciones, al establecimiento de especies con requerimientos de sitios más exigentes, tales como lenga en suelos carentes de cubierta vegetal o de especies tolerantes en suelos perturbados.

En las provincias de Coyhaique y General Carrera las probabilidades de éxito son mayores en las partes altas de las cuencas hidrográficas (altitud superior a los $500 \mathrm{msnm}$ ) y en la vertiente oriental de la cordillera de Los Andes, con suelos algo desarrollados y medianamente profundos

\section{DISCUSIÓN}

La actividad forestal en Chile se ha desarrollado como un sistema de producción sin integración a la actividad agrícola y ganadera. Los paquetes tecnológicos han consistido en 
modelos productivos monoespecíficos que requieren de fuertes concentraciones de tierra, capital y tecnología, sin soluciones mixtas o forestalmente integradas. Los sistemas de plantación, así como los esquemas de manejo existentes, se ajustan por lo tanto a plantaciones masivas orientadas a la producción a gran escala, modelo de difícil réplica a escala pequeña e inadecuada a las necesidades y posibilidades del sector campesino de la región de Aysén.

En efecto, desde la promulgación del DL 701 en el año 1974, en la región de Aysén se han establecido cerca de 45 mil hectáreas de plantaciones. De ellas sólo un poco más de 30 mil han sido acreditadas; es decir, en promedio 850 hectáreas por año. Para una región con casi 1 millón de hectáreas en diferentes estados de erosión y sin cubierta vegetal (CONAF - CONAMA, 1999), de las cuales 500 mil corresponderían a suelos productivos (Moreno, 2008), se requerirían 700 años para forestarlas. Otra paradoja es que el 99\% son plantaciones monoespecíficas con objetivos de protección o producción de madera y el 99,7\% corresponde a plantaciones con especies exóticas.

La importancia de las plantaciones multipropósitos radica en su habilidad para aumentar la productividad, permitiendo que el rendimiento del suelo no se vea disminuido en el curso de los años. Además de ello, son sistemas que permiten recuperar áreas degradadas y con conflictos de usos. Integrar árboles, cultivos agrícolas y ganado crea un sistema de manejo del suelo para generar productos comerciales mientras se mantiene productividad a largo plazo. Se reduce el riesgo económico porque el sistema ofrece productos múltiples, la mayoría de los cuales tienen un mercado establecido. Se reducen los costos de producción y la flexibilidad de la comercialización es realzada distribuyendo costos del manejo entre la madera, los componentes del ganado y los productos agrícolas (Klopfenstein et al., 1997).

A partir de lo anteriormente expuesto, se puede indicar cuáles son las principales ventajas de las plantaciones multipropósitos, que hacen de ellas un instrumento de desarrollo adecuado para las comunidades campesinas de la región. Las ventajas de los sistemas mixtos en comparación con los sistemas basados en monocultivo se exponen a continuación:

Micro-ambiente favorable para los animales: El micro-clima que se crea bajo los árboles beneficia también a los animales domésticos que se mantienen más frescos a la media sombra que bajo el fuerte sol. Aunque en las condiciones de pastoreo en praderas artificiales, los bovinos tienden a pastar preferiblemente en las horas más frescas, ciertamente su consumo se ve limitado tanto por razones de regulación del balance térmico como por restricciones del horario de pastoreo.

Expansión de la Biodiversidad: Estas plantaciones establecidas para la producción multipropósito también favorecen gradualmente el aumento la biodiversidad de especies animales silvestres, y la recuperación de los nutrientes presentes en la vegetación original a partir de la extracción de los mismos del subsuelo.

Potencial de Fijación de Carbono: Aunque recientemente se ha calculado el potencial de fijación de carbono atmosférico en praderas con pastos mejorados, la capacidad de fijar carbono, y con esto contribuir a reducir el efecto invernadero, de los sistemas mixtos (silvopastorales) es significativamente superior a cualquier monocultivo. 
Ventajas adicionales a nivel de predio: La introducción de especies arbórea en el predio proporcionan beneficios adicionales como protección de mini-cuencas, incluyendo la protección del suelo contra la erosión principalmente en áreas de pendiente; producción de postes, estacas y madera; protección del viento; y embellecimiento del entorno.

Beneficios económicos: Productores o agricultores obtienen beneficios económicos que satisfacen sus necesidades de leña, postes, varas, madera de aserrío, frutas, alimento para el ganado, flores para miel, productos medicinales, otros.

Reducen la dependencia asociada a monocultivos: Problemas originados en regímenes pluviométricos irregulares, fluctuaciones de mercado, propagación de plagas; dificultad para adquirir plaguicidas, fertilizantes, maquinaria o repuestos, concentrados para ganado, etc. que con frecuencia suben su costo drásticamente.

Flujos de caja: Las inversiones económicas asociadas al establecimiento de árboles cosechables pueden aminorarse considerablemente gracias a los beneficios de cultivos anuales durante los primeros años de crecimiento de los árboles. En algunos casos, puede aumentar el número de años asignados para estos cultivos, por medio de raleo, poda o manipulación de copas superiores, de modo que también se pueden obtener beneficios económicos adicionales (postes, leña) en los primeros años de desarrollo de los árboles.

Control e malezas: La presencia de árboles usualmente disminuye los costos de control de malezas; además pueden emplearse para cercas y otros mecanismos preventivos contra la usurpación de tierras.

Distribución del trabajo: Se flexibiliza la distribución de la carga de trabajo durante el curso del año y se utiliza mano de obra local.

Mejoramiento de suelos: Los árboles aportan nutrientes mediante la materia orgánica del follaje, el ganado incorpora estiércol y hay fijación de nitrógeno cuando se usan leguminosas.

Obviamente hay un campo considerablemente amplio para mejorar, por medio del diseño, otros sistemas más productivos y con mayores rendimientos, asociando especies más deseables de plantas y animales, en espacio y tiempo, y basados en la experiencia local y mundial, estabilizando los patrones de la agricultura de subsistencia, al promover la diversificación de cultivos agrícolas asociados a especies arbóreas.

La transición desde un modelo industrial monoespecífico a otro mixto y con pertinencia regional, debe ser viable y sostenida, para esto los protagonistas no pueden ser los organismos públicos, tampoco los iniciados o interesados en el tema como forestales, ecólogos, voluntarios externos, tampoco las universidades o centros especializados, sino los propios campesinos, en el entendido que el problema de la participación es determinante y no sólo un componente folclórico del desarrollo rural. 


\section{CONCLUSIONES}

Existen especies nativas, que no crecen en forma natural en la región, que se adaptan fácilmente a las condiciones ambientales. Araucaria ha demostrado ser una especie que se adapta bien a los requerimientos de los campesinos de la región, por cuanto no requiere de exclusión de animales para su establecimiento y desarrollo posterior. Por tanto su establecimiento no es incompatible con la ganadería.

El mucrón coriáceo de las hojas reprime el ramoneo de ovinos y bovino de las plantas de araucarias. En el caso de caballares y caprinos, éstos ramonean las plantas de araucarias y por tanto no es recomendable introducirlas en etapas tempranas de la plantación

El único daño reportado es por pisoteo y según los propietarios entrevistados, este se produce durante los dos primeros años de la plantación.

En condiciones de suelos profundos y bien drenados, araucaria puede crecer a tasas anuales promedios de $1 \mathrm{~cm}$ de diámetro y 0,5 metros en altura. Por lo que no es ilusorio pensar en rotaciones de 80 a 100 años con diámetros superiores a $50 \mathrm{~cm}$ y alturas de 30 a $40 \mathrm{~m}$.

El crecimiento en forma natural de radal en suelos de la comuna de Lago Verde, supera los $11 \mathrm{~m}^{3} / \mathrm{ha} /$ año. Superior en casi un $100 \%$ al crecimiento de bosques de lenga o especies del Tipo Siempreverde.

La producción de biomasa en radal se concentra en los primeros 20 años, después decae y comienza a ser reemplazado por especies más exigentes en cuanto a condiciones de sitios.

El rápido crecimiento inicial de radal, unido a la habilidad de rebrotar de tocón, le permite establecerse exitosamente incluso en presencia de animales domésticos del tipo bovino.

Araucaria, puede proveer madera de alta calidad, frutos, y abrigo al ganado bajo un modelo mixto silvopastoral.

Radal puede proveer leña, abrigo al ganado y protección al suelo, además de facilitar el acceso de especies exigentes y de mayor interés desde el punto de vista maderero o de la restauración ecológica. 


\section{REFERENCIAS}

Cisternas, J. C., Solís, R., 2009. Propuestas de Manejo para el Radal en la Comuna de Lago Verde. Documento Técnico. Corporación Nacional Forestal, Región de Aysén. 12 páginas

CONAF/CONAMA, 1999. Catastro y Evaluación de Recursos Vegetacionales Nativos de Chile. Informe Nacional con variables ambientales. Región de Aysén. Santiago. Chile. 90 pp.

Cortes, M., 2003. Dinámica y Conservación de Araucaria araucana en la Cordillera de la Costa de Chile. Tesis Facultad Ciencias Forestales Universidad Austral de Chile. Valdivia. 112 páginas

Del Fierro, P.; Pancel, L.; Rivera, H.; Castillo, J., 1998. Experiencias Silviculturales del Bosque Nativo Chileno. Recopilación de Antecedentes para 57 Especies Arbóreas y Evaluación de Prácticas Silviculturales. PCMSBN. CONAF. Santiago. Chile. 420 páginas

Donoso, C., 2006. Las especies Arbóreas de los Bosques Templados de Chile y Argentina. Autoecología. Marisa Cuneo Ediciones. Valdivia. Chile. 678 páginas

Donoso, Claudio, 1993. Bosques Templados de Chile y Argentina. Editorial Universitaria. Santiago. Chile.483p

Donoso, C.; Gerding, V.; Olivares, B.; Real, P.; Sandoval, V.; Shlatter, R. y Schlegel, F., 1984. Antecedentes para el Manejo del Bosque Nativo de Forestal Arauco (Sector Cordillera de Nahuelbuta). Informe de Convenio N 74. Universidad Austral de Chile. 183 páginas.

Di Castri, F. y Hajek, E., 1976. Bioclimatología de Chile. Imprenta-Editorial de la Universidad Católica de Chile. Santiago. 129 páginas

Moreno, P., 2008. Aysén: Perspectivas y Desafíos para un Uso Sustentable. En Seminario Bosques Nativos: Oportunidades para el Desarrollo Rural. Instituto Forestal. Valdivia. Chile

Klopfenstein, N; Rietveld, W. y Carman, R., 1997. Silvopastoreo: An Agroforestal Practice USDA Forest Service, Rocky Mountain Research Station, National Agroforestal Center, East Campus - UNL, Lincoln. Disponible en: http://www.unl.edu/nac/pubs/afnotes/sil-1/index.html

Kurtz, V.; Ferruchi, M.; Marecos, V. y Bullman, A. 2003. Comparación entre Diferentes Técnicas de Establecimiento de Plantaciones de Araucaria angustifolia, en Misiones, Argentina. Novenas Jornadas Técnicas Forestales. INTA-FCF-MEYRNRYT-El Dorado, Misiones, Argentina

Veblen, T., 1982. Regeneration Patterns in Araucaria araucana Forest of Chile. Journal of Biogeography 9:11-28.

Veblen, T.; Burns, B.; Kitzberger, T.; Lara, A. and Villalba, R., 1995. The Ecology of the Conifers of Southern South America. In.: Enright N \& R. Hill (Eds). Ecology of the Southern Conifers. 120-155 p.

Wilhelm de Mösbache, E., 1999. Botánica Indígena de Chile. Primera Edición. Editorial Andrés Bello. Santiago. Chile. 140 Páginas 
